\title{
The Effect of Natural Convection on Equiaxed Dendritic Growth: Quantitative Phase-Field Simulation and Comparison with Synchrotron X-Ray Radiography Monitoring Data
}

\author{
Xin Bo Qi, Yun Chen, Xiu Hong Kang, and Dian Zhong Li \\ Shenyang National Laboratory for Materials Science, Institute of Metal Research, Chinese Academy of Sciences, \\ Shenyang, Liaoning 110016, China
}

Correspondence should be addressed to Yun Chen; chenyun@imr.ac.cn

Received 18 May 2016; Accepted 30 August 2016

Academic Editor: Paolo Ferro

Copyright (C) 2016 Xin Bo Qi et al. This is an open access article distributed under the Creative Commons Attribution License, which permits unrestricted use, distribution, and reproduction in any medium, provided the original work is properly cited.

\begin{abstract}
A two-dimensional (2D) quantitative phase-field model solved by adaptive finite element method is employed to investigate the effect of natural convection on equiaxed dendritic growth of $\mathrm{Al}-4 \mathrm{wt} . \% \mathrm{Cu}$ alloy under continuous cooling condition. The simulated results are compared with diffusion-limited simulations as well as the experimental data obtained by means of in situ and realtime X-ray imaging technique. The results demonstrate that natural convection induced by solute gradients around the dendritic crystal has an obvious influence on the dendrite morphology and growth dynamics. Since the rejected solute cooper from solid is heavier than aluminum, it sinks down along the interface from the top arm tip to the bottom arm which results in the formation of a circulatory flow vortex on both sides of the dendrite. Hence, the convection promotes the top arm advancing into the melt progressively whereas it suppresses the growth of bottom severely. As the dendrite grows into a large size, the convection becomes more intense and the morphology shows distinguished asymmetric shape. When compared with experimental data, the growth velocity is found to agree substantially better with the simulation incorporating natural convection than the purely diffusive phasefield predictions.
\end{abstract}

\section{Introduction}

Equiaxed dendritic crystal is one of the most common microstructures formed in the solidification process of materials, whose morphology, size, and composition distribution in castings are critical to the mechanical properties of the as-cast structural materials. Ever better understanding of its morphology evolution dynamics and related underlying physics are always important to obtain targeted grain features as well as deepen the knowledge of formation mechanism on such practical and theoretical important structure. Since many factors, including the characteristics of the alloy system (diffusion, anisotropy, melting point, etc.) and the external imposed conditions of solidification (composition, cooling rate, thermal gradient, undercooling, forced flow, etc.), exert strong influences on the growth shape and dynamics of equiaxed dendrites, it is of great importance to discriminate these factors on the growth behaviors $[1,2]$.
As one of the factors impacting the dendrite growth dynamics, fluid flow, in particular the natural convection which is caused by the density variation in the melt, is one of the main driving forces to form various morphologies of individual dendrite in the real solidification conditions $[3,4]$. It alters the local distribution of heat and solute around the dendritic tip. Nevertheless, natural convection is unavoidable on the earth because of the inhomogeneous distribution of solute and heat that results in the expansion or compression of melt. Thus, the gravity drives the denser melt moving downwards and the lighter melt flowing upwards, provoking the convective flow of melt. Consensus has been already achieved that natural convection should be responsible for the deviation between experiments and theoretical predictions through plenty of experimental analyses on the dendritic growth of transparent organic alloys [5, 6]. For example, the comparison of the SCN crystals grown in the microgravity environment which was generated by the 
space shuttle Columbia [7-9] with that under terrestrial conditions performed by Glicksman et al. demonstrated that the gravity-driven convection is the main factor contributing to the deviation between the measured dendritic growth velocities and the classical diffusion-limited Ivantsov theory [10]. Despite the fact that some reliable conclusions have been drawn on the solidification of transparent organic alloys, there have been limited experiments on metallic systems. Moreover, it is well known that the solidification behavior of transparent alloys differs from that of metallic systems owing to their different thermophysical properties. And these previous experiments cannot illustrate the dynamic details of morphology evolution precisely, especially the distribution of solute. In recent years, synchrotron X-ray radiography has become an effective method to unveil the dynamical formation of solidification microstructures and grain structure in metallic alloys. Benefitting from this development, precise data by in situ and real-time observations are obtained to benchmark modeling and numerical simulations [11-17]. Bogno et al. $[13,16]$ have carried out a series of in situ and real-time observations of the equiaxed dendritic growth of Al-Cu alloys solidified by cooling-down at the European Synchrotron Radiation Facility (ESRF). They have carefully characterized and analyzed the dendritic growth velocity and the solute distribution among the dendritic grains. Nevertheless, flow effects on dendritic growth dynamics were not taken into account in their analysis because of the difficulty to estimate the natural convection. Through comparing the diffusion-based phase-field simulations with the measured characteristic growth parameters of equiaxed dendritic crystal on Al-4wt.\%Cu alloys, Chen et al. [16] have found that the gravity-driven melt convection plays an important role on the crystal growth in the in situ and real-time observed experiment. The transportation of solute by convection results in the pronounced discrepancy in the dynamics of equiaxed grain growth between experiment and quantitative simulations.

Besides experimental approaches, theoretical analysis and numerical computer simulations have also been exerted to understand the natural convection effects on dendritic crystal growth. Yet, analytic solutions which require lots of assumptions beforehand [18-23] are limited to incorporate the complex nonlinear effect of convection into the theoretical analyses of the dendritic growth. Additionally, these analytic solutions are not able to give the details of the morphology evolution of a dendrite and the flow pattern in the melt. In contrast, several numerical mathematical models, such as phase-field and front-tracking methods, have been extended to include fluid flow dynamics for dendritic growth, offering researchers a rather convenient way to investigate the melt convection effects [24-29]. Bänsch and Schmidt [30] presented a numerical algorithm based on a sharp-interface model to simulate the thermal convection with different boundary conditions such as Dirichlet and Neumann boundary conditions. Tönhardt and Amberg [31] simulated the natural convection on succinonitrile (SCN) using a two-dimensional phase-field model, which revealed that the influence of thermal natural convection increases with the growth of the dendrites. Zhao et al. [32] presented the simulation using a sharp-interface model and adaptive mesh technique with the data extracted from Tonhardt's work and obtained the similar values and conclusions. Chen and Lan [33] used an efficient adaptive three-dimensional phasefield model to investigate the influence of thermal convection on SCN dendrite. Their simulation results are consistent with previous theories and experimental observations.

In this paper, the equiaxed dendrite growth from the isothermal melt cooled by a constant rate on $\mathrm{Al}-4 \mathrm{wt} . \% \mathrm{Cu}$ alloy is simulated with the employment of a $2 \mathrm{D}$ quantitative phase-field model with incorporation of incompressible Navier-Stokes equations. The simulated natural convection and its evolution with time, as well as the dendritic growth dynamics, are characterized and analyzed in detail. And then the simulations were directly compared with diffusionlimited dendritic growth and the monitored data of the alloy by in situ and real-time X-ray radiography [16]. Within the comparison results, the effects of natural convection on equiaxed dendritic growth dynamics and morphology are thus clarified, and it is clearly demonstrated that natural convection is an important factor accounting for the discrepancy between predictions controlled by diffusion and experimental data.

\section{Phase-Field Model and Numerical Implementation}

2.1. Model Description. The phase-field model for directional solidification [34] is modified and adapted to simulate equiaxed dendritic growth under continuous cooling-down condition. This model is primarily proposed by Karma [35] where the solute antitrapping current is included in the solute conservation equation to eliminate the unphysical effects, such as the surface diffusion, surface stretching, and the jumping of chemical potential at the interface. In the employed model, the order parameter $\phi$ is defined as 1 in solid and -1 in liquid and changes smoothly at the interface layer. The equation for phase-field variable is given by

$$
\begin{aligned}
{[1} & \left.-\frac{R t}{m c_{l}^{0}}\right] \tau_{0} a_{s}^{2}(\vec{n}) \frac{\partial \phi}{\partial t} \\
= & \nabla\left[W_{0}^{2} a_{s}^{2}(\vec{n}) \nabla \phi\right]-\frac{\partial}{\partial x}\left[W_{0}^{2} a_{s}(\vec{n}) a_{s}^{\prime}(\vec{n}) \frac{\partial \phi}{\partial y}\right] \\
& +\frac{\partial}{\partial y}\left[W_{0}^{2} a_{s}(\vec{n}) a_{s}^{\prime}(\vec{n}) \frac{\partial \phi}{\partial x}\right]+\phi-\phi^{3} \\
& -\lambda\left(1-\phi^{2}\right)^{2}\left[U-\frac{-R t}{(1-k) m c_{l}^{0}}\right]
\end{aligned}
$$

where $R$ is the cooling rate, $c_{l}^{0}$ is the initial concentration of the alloy, and $W_{0}$ and $\tau_{0}$ are the interface width and relaxation time, respectively. $\lambda$ is the coupling parameter between concentration field and phase field, expressed as $\lambda=a_{1} \xi=a_{1} W_{0} / d_{0}$. The chemical capillary length is $d_{0}=\Gamma /|m| c_{0}(1-k)$ with $m$ being the liquidus slope and $k$ being the solute partition coefficient, which are taken constants, and $\Gamma$ being the Gibbs-Thomson coefficient. As it 
has been demonstrated in the reference [16], the capillary length $d_{0}$ is taken as constant even though the temperature of the melt decreases with time. $U$ is the dimensionless solute concentration; $U=\left(2 c / c_{0}-[1+k-(1-k) \psi]\right) /(1-k)[1+k-$ $(1-k) \psi]$, which is obtained by solving the solute conservation equation that is written including the terms describing the solute transport by convection:

$$
\begin{aligned}
& {[1+k-(1-k) \phi] \frac{\partial U}{\partial t}+\left(\frac{1-\phi}{2}\right) \vec{v}} \\
& \cdot\{[1+k-(1-k) \phi] \nabla U-[1+(1-k) U] \nabla \phi\} \\
& =\nabla \\
& \quad \cdot\left\{D(1-\phi) \nabla U+\frac{1}{\sqrt{2}}[1+(1-k) U] \frac{\partial \phi}{\partial t} \frac{\nabla \phi}{|\nabla \phi|}\right\} \\
& \quad+[1+(1-k) U] \frac{\partial \phi}{\partial t},
\end{aligned}
$$

where $k$ is the solute partition coefficient and $D$ is the solute diffusion coefficient in liquid. $W_{0}$ and $\tau_{0}$ are the length scale and time scale, respectively.

Then, the fluid flow is coupled using the method proposed by Beckermann and coworkers [36]. A no-slip condition at the solid-liquid interface is imposed by adding a source term in the Navier-Stokes equations. Assuming the fluid is incompressible, the Navier-Stokes equations controlling the fluid motion are rewritten as follows:

$$
\begin{aligned}
& \frac{\partial(1-\phi) \vec{v}}{\partial t}+(1-\phi)(\vec{v} \cdot \vec{\nabla}) \vec{v} \\
& =-(1-\phi) \vec{\nabla} \frac{p}{\rho}+\vec{\nabla}[v \vec{\nabla}(1-\phi) \vec{v}]+M_{l}^{d}+B_{i}, \\
& B_{i} \\
& = \begin{cases}0, & i=x, \\
(1-\phi) g\left[1-\alpha_{T}\left(T-T_{0}\right)+\alpha_{c}\left(c-c_{0}\right)\right], & i=y,\end{cases} \\
& M_{l}^{d}=-h v \frac{(1+\phi)^{2}(1-\phi)}{2 W_{0}^{2}} \vec{v},
\end{aligned}
$$

where $\vec{v}$ is the liquid flow velocity, $v$ is the liquid kinematic viscosity, $p$ is the pressure in the melt, and $\rho$ is the density. The term $M_{l}^{d}$ is a dissipative interfacial force to constrain the flow velocity to be zero in the bulk solid phase. Physically, in the limit of a sharp interface, that is, $W_{0} \rightarrow 0$, the liquid velocity has to approach 0 in order to generate a moderate drag force as illustrated in the expression of $M_{l}^{d}$; as a result, the usual no-slip condition at the solid/liquid interface is resumed. Then, the exact value of $h$ is determined from an asymptotic analysis to minimize the deviations between the diffuse-interface and sharp-interface solutions $[37,38]$. The buoyancy force $B_{i}$ which depends on the differences of local temperature and composition is expressed using the
Boussinesq approximation. The conservation equation for mass takes the following form:

$$
\nabla \cdot\left[\frac{(1-\psi)}{2} \vec{v}\right]=0 .
$$

2.2. Numerical Simulation. The governing equations of the model, (1)-(4), are numerically solved to simulate equiaxed dendritic growth of $\mathrm{Al}-4 \mathrm{wt} . \% \mathrm{Cu}$ alloy under continuous cooling-down condition. In order to increase computational efficiency, the adaptive finite element method is adopted, where the meshes are coarsened or refined according to the local error indicator [39]. The numerical implementation is based on the finite element package AFEPack [40]. The error indicator is based on the gradient jump of finite element solutions on the interface of adjacent elements. Here the error indicator is chosen as

$$
\eta=\sum_{e \in \partial T} \int_{e} \nabla \phi_{h} \cdot n_{e} d \Gamma
$$

where $\phi_{h}$ is the finite element solution for the phase-field equation and $\partial T$ is the boundary of the element $e$. The parameters used in this study are listed in Table 1, and the cooling rate $R$ is set equal to that applied in the experiment.

The simulation domain is rectangle with a size of $4304 \mu \mathrm{m}$ $\times 2152 \mu \mathrm{m}$. A nucleus is placed in the center of the domain with its preset crystallographic orientations aligned to the coordinate axes. Zero-flux Neumann boundary conditions are imposed on the boundaries for both phase-field and solute conservation equations. The initial conditions are as follows: the nucleus is assumed to be circular with a nondimensional radius $r_{0}$ of 10 ; the phase-field variable is initialized as $\phi(r, 0)=-\tanh \left[\left(r-r_{0}\right) / \sqrt{2}\right]$; the initial concentrations (dimensionless) are set to zero in both liquid and solid. The adaption in the present work is thus accomplished by making a coarse initial mesh and then adaptively splitting or merging the elements according to the error indicator mentioned above.

\section{Results and Discussion}

3.1. Simulation Results of Phase-Field Model. Figure 1 shows the flow field around the crystal at different solidification time, together with the solutal field. It can be seen that, with continuous cooling of the melt, the seed starts to grow and the solute is rejected from the crystal (the partition coefficient is smaller than one), leading to the increment of the liquid density around the seed. The enriched copper liquid, which is heavier than aluminum, sinks down along the solid crystal boundaries, whereas the melt far away from the crystal moves upwards due to the lower density. As a result of this motion, a convective vortex initializes on both sides of the crystal at the middle part of the domain. And the maximum fluid velocity appears on the centerline just below the crystal because of the almost mirror symmetrical shape of the dendrite. The flow flux in the vicinity of the dendrite is always downwards, which transports the solute from upper region to lower place. Therefore, the solute accumulates at the lower part of the 

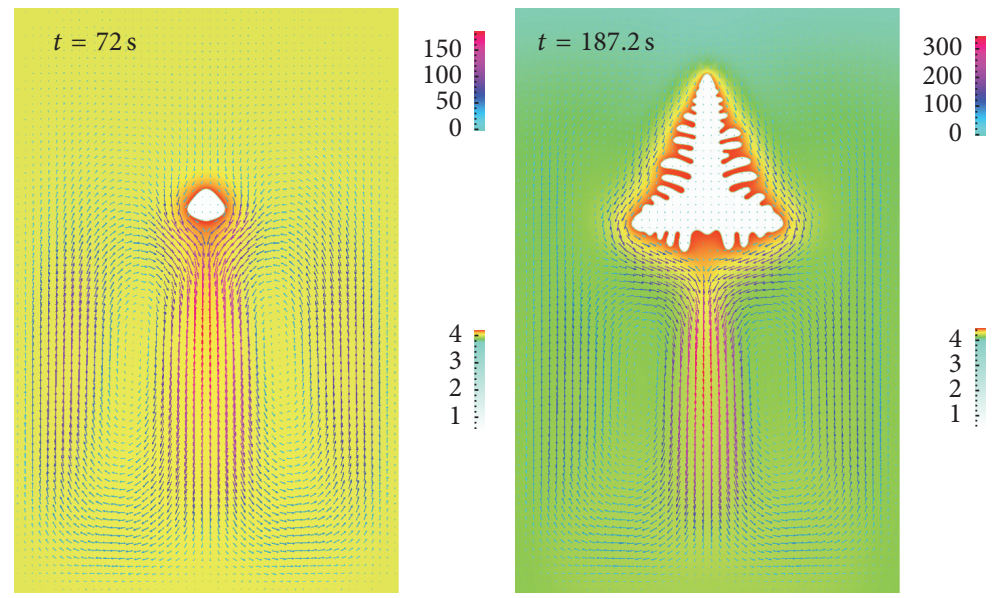

FIGURE 1: Two snapshots of the simulated flow field and solutal field for $t=72 \mathrm{~s}$ and $187.2 \mathrm{~s}$. The upper legend is for flow velocity with units of $\mu \mathrm{m} / \mathrm{s}$ and the lower legend is the weight percent of solute $\mathrm{Cu}(\mathrm{wt} . \%)$.

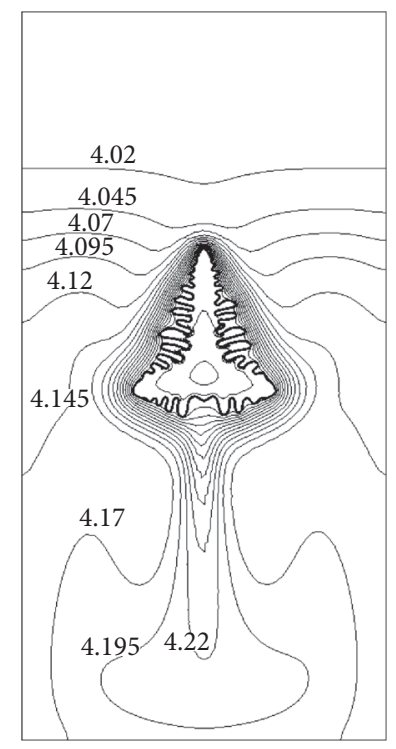

(a)

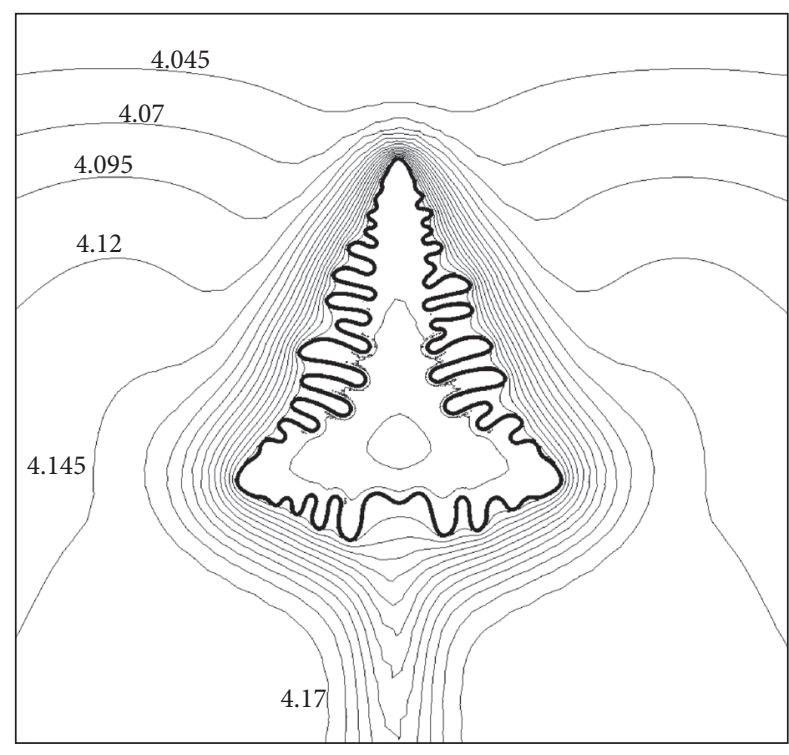

(b)

FIGURE 2: Isoconcentration contours around a dendrite with developed side branches. (a) The whole domain. (b) Close-up around the crystal.

melt, around the bottom arm tip. In turn, the accumulation of solute increases the melt convective strength below the dendrite. Hence, the centers of the two vortices move to a lower and lower position with solidification proceeding until it is prevented by the domain wall. When examining the maximum convective velocity, it appears that, at the early stage of dendritic growth, the natural convection is relatively weak and the maximum fluid velocity which occurs right under the crystal between the two vortices is $21.52 \mu \mathrm{m} / \mathrm{s}$ at $t=14.4 \mathrm{~s}$. The fluid in the vortices continues accelerating as the dendrite grows and the maximum fluid velocity is $342.71 \mu \mathrm{m} / \mathrm{s}$ at $t=187.2 \mathrm{~s}$.

Figures 2(a) and 2(b) show the isoconcentration field in the entire domain and in the vicinity of the crystal, respectively. It can be seen that natural convection significantly influences the shape of the dendrite and the concentration field. The isolines of solute concentration are compressed near the top tip interface and stretched around the bottom tip by the descending flow. This situation is similar to the simulation of dendrite growth of pure substance under force flow [25]. At the region above the solute boundary layer of the top interface, the concentration field is hardly disturbed by the flow; on the contrary, below the dendrite it is strongly affected by the flow. The tips growing in the upward, downward, and horizontal directions are referred to as the upstream tip, downstream tip, and perpendicular tip, respectively. The time-dependent arm lengths of these three tips are plotted in Figure 3. At the early stage of solidification, the natural convection is so weak that its influence on dendrite growth is not obvious as indicated by the coincide curves. Gradually, the difference between the upstream and the downstream tip becomes more and more noticeable. The 


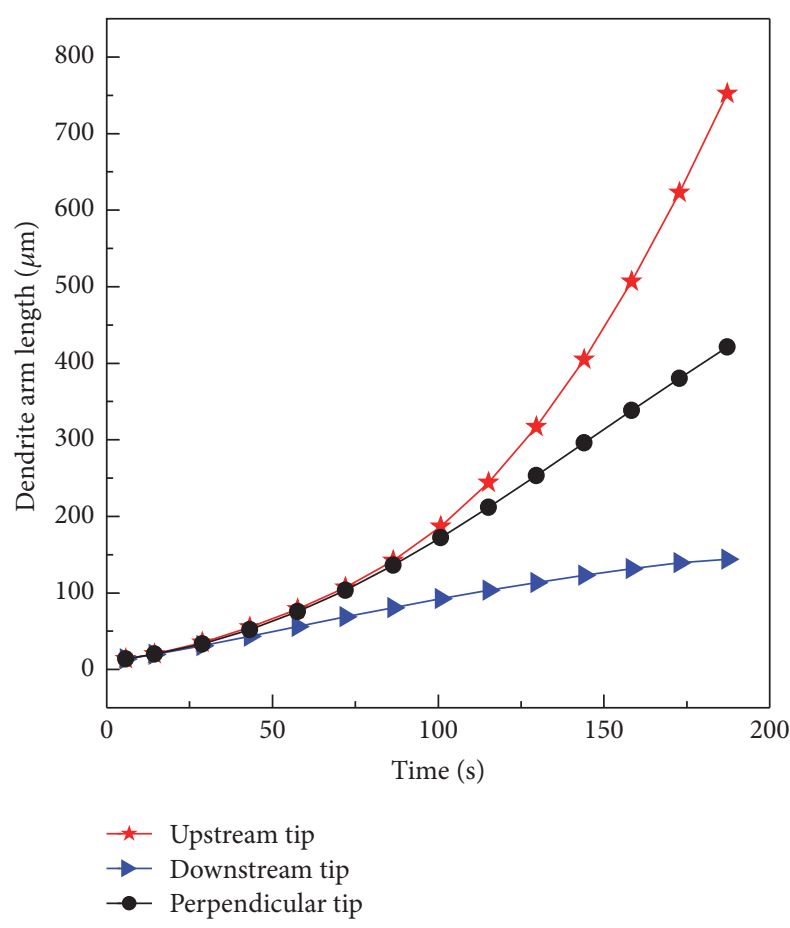

FIGURE 3: The primary-dendrite arm length with time in the phasefield simulation.

length of the upstream arm is much longer than those of the downstream tip and perpendicular tip at the final stage of the simulated solidification. This is because the strong natural convection which takes the rejected solute away adequately at the upstream tip promotes its growth. Conversely, the downstream tip is retarded, which suffers not only from its own rejected solutes, but also from other solutes carried by the convection. An interesting phenomenon is that the downstream tip even disappears finally as it is stifled by two neighbor side branches. For the perpendicular tip, it grows slightly downwards, because the natural convection imposes a downward force on it.

The measured lengths in Figure 3 show that the primary arms of the dendrite all keep increasing with time. The arms exhibit almost identical advancing rate at the early stage $(t<50 \mathrm{~s})$, and then the growing speeds of upstream and downstream arm tip deviate from each other apparently. In order to quantitatively describe the effect of convection on the dendrite growth morphology, the dendritic arm length ratio is then adopted to evaluate the asymmetry of the dendrite shape and defined as follows:

$$
r=\frac{l_{\text {upstream }}}{l_{\text {downstream }}},
$$

where $l_{\text {upstream }}$ and $l_{\text {downstream }}$ are the lengths of the upstream arm and the downstream arm, respectively. The length ratio evolving with time is plotted in Figure 4(a). It increases monotonically with time, which indicates that as the dendrite grows into a larger size, the difference between the two arms becomes more pronounced. The deviation of the ratio from units represents the asymmetry growth of the dendrite. And the larger the ratio deviates from unity, the stronger the natural convection around the dendrite is, just as evidenced in Figure 4(b) which plots the variation of the maximum magnitude of the flow field with the dendrite arm length ratio.

To be more exact, as the dendrite grows, more solute will be rejected into the melt ahead of the solid/liquid interface. Meanwhile, the region of melt with different density caused by the rejected solute becomes wider and wider. In terms of the dimensionless Rayleigh number which indicates the strength of nature convection, the convective strength enhances notably by the expanded region of distribution of solute. Therefore, stronger convection forms around the dendrite. The pronounced influence of natural convection on solid growth dynamics can also be understood within the order of magnitude differences between the moving velocities of the tip front and the melt. At the end of the calculation, the maximum flow velocity is $342.71 \mu \mathrm{m} / \mathrm{s}$, while the upstream tip velocity and the downstream tip velocity are $8.98 \mu \mathrm{m} / \mathrm{s}$ and $0.33 \mu \mathrm{m} / \mathrm{s}$, respectively (more details about the tip velocity are shown below). This means that the flow velocity can be hundreds of times faster than the tip velocity. Hence, the natural convection induced by a dendrite itself plays an important role in the dendritic growth behavior.

Figure 5 presents the evolution with time of the tip velocities for the three arms. As mentioned above of the dendrite arm length evolution, the growth velocity of the upstream tip is the highest which continuously increases with time. This can be ascribed to two aspects: (i) the melt is exposed to an ex ternal cooling-down condition, which keeps providing additional driving force for crystallization; and (ii) the convective flow takes the rejected solutes away adequately. Both effects lead the upstream tip to grow at a higher speed. The growth velocity of the perpendicular tip increases at the early stage and ceases to reach a steady state after a long time. Although the perpendicular tip is under the same imposed cooling condition as the upstream tip, it seems that the external coolingdown does not boost its growth velocity when compared to the diffusion-limited growth velocity [16]. The explanation may be that the convection of the solute from the upstream portion of the dendrite retards the perpendicular tip. In other words, the natural convection counterbalances the driving force for the migration of tip front caused by the increased thermal undercooling of bulk melt. The behavior of the downstream tip is similar to the perpendicular one. But one difference should be emphasized that the former arm growth velocity intends to decrease with time, even to zero at the end of simulation while the latter remains almost unchanged. The reason for this difference can be attributed to the morphology of the dendrite, with two neighbor side branches stifling the growth of the downstream tip.

Furthermore, the evolution of the tip shape is examined of the three tips through measuring the radii as illustrated in Figure 6. It is surprising that, for each tip, the tip radius increases with the applied external undercooling at the early stage of solidification and then it reaches a peak value. Finally, the radii continue decreasing with undercooling. The increment of tip radii with undercooling is contrary to the classical solidification theories, $r^{2} V=\mathrm{Ct}$, where $r$ is the tip 


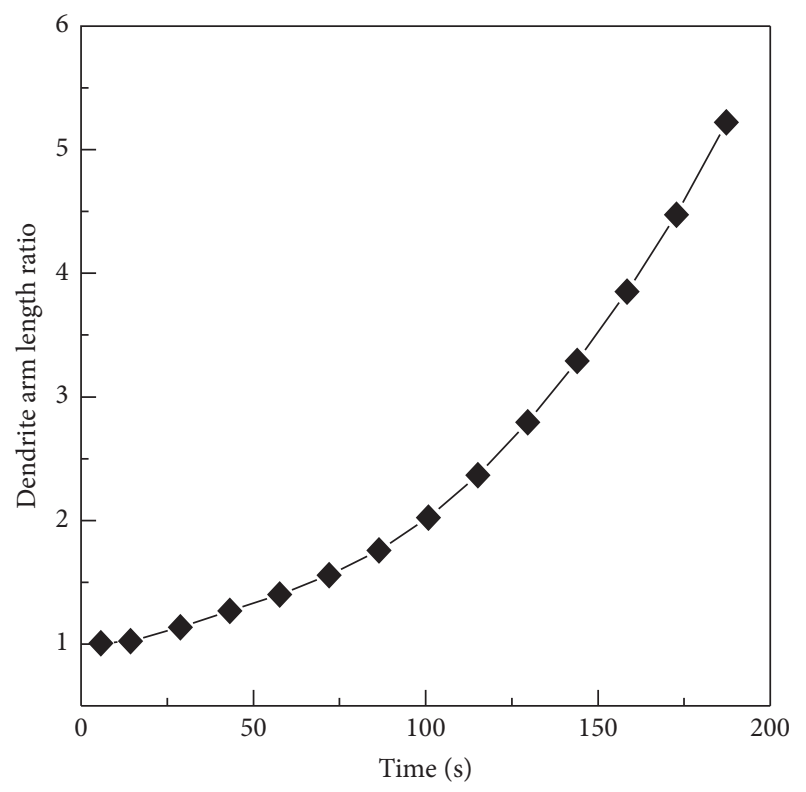

(a)

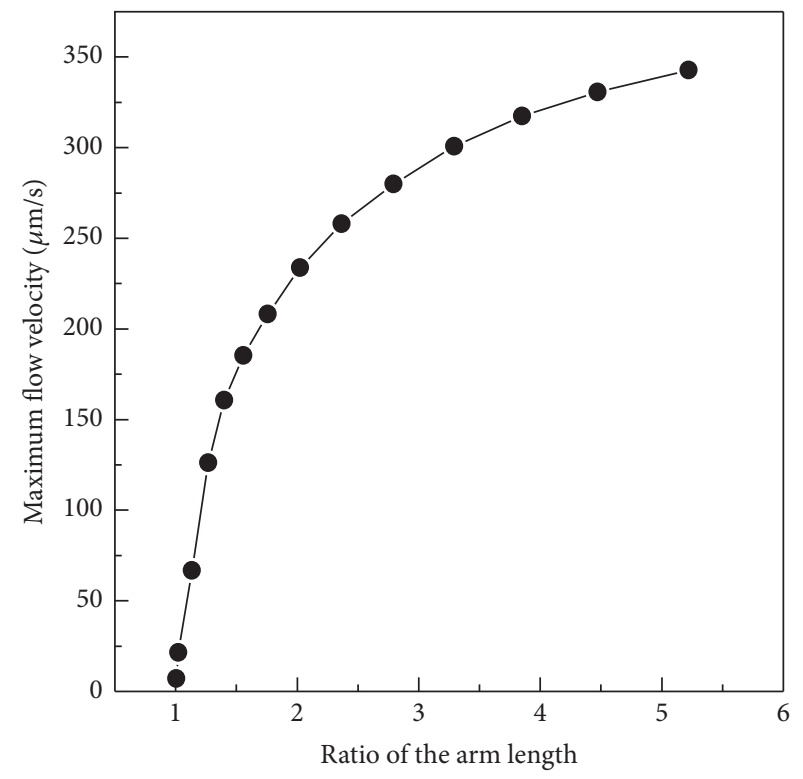

(b)

FIGURE 4: The growing dendrite leading to the increment of natural convection which in turn results in the asymmetry of the dendrite shape. (a) The variation of dendrite arm length ratio with time. (b) The relationship between the maximum flow strength and the dendrite arm length ratio.

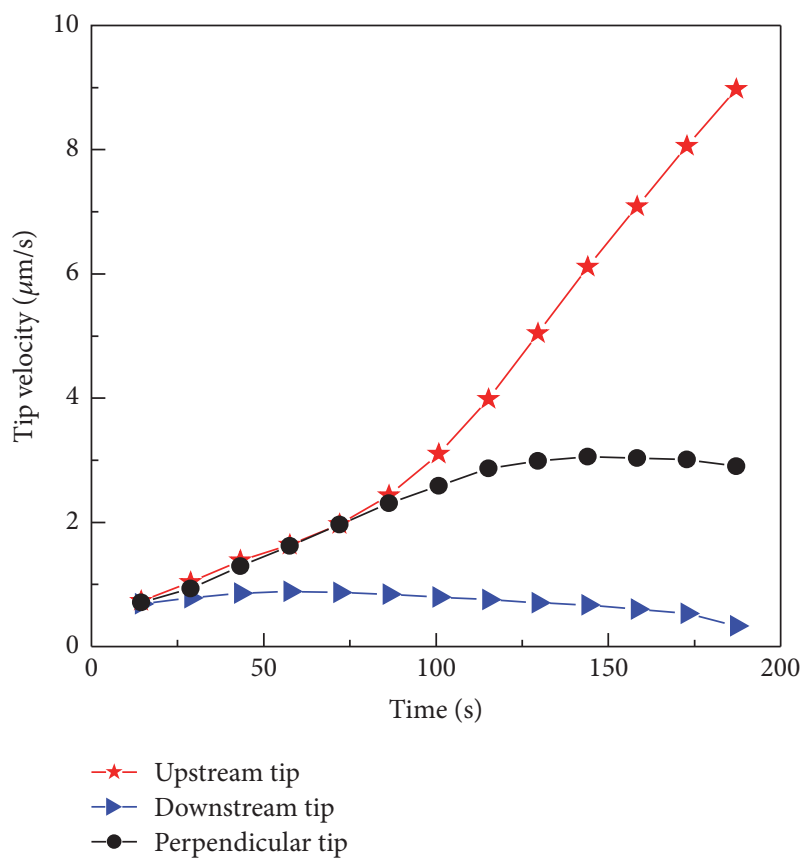

FIGURE 5: Evolution of the dendrite tip velocities of three different tips in the phase-field simulation.

radius, $V$ is the tip velocity, and $\mathrm{Ct}$ is a constant value which depends on the material. This inconsistence with theoretical predictions may be linked to the fact that the crystal is still a globular grain at the early stage. The theoretical relationship derived from the morphology of dendrite with parabolic tip

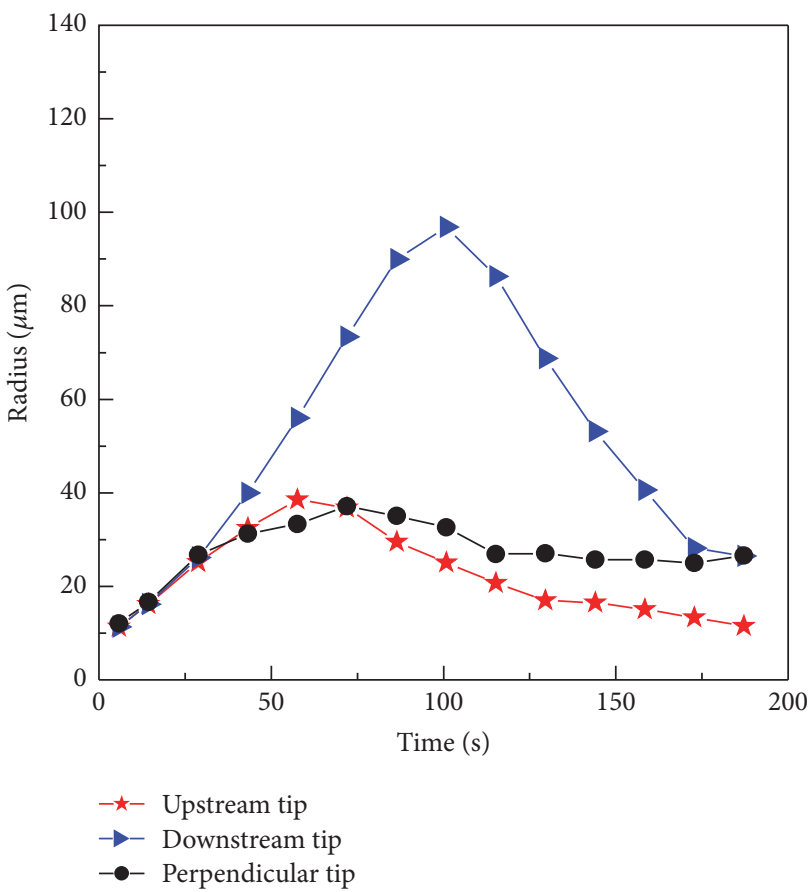

FIGURE 6: Evolution of the dendrite tip radii of three different tips in the phase-field simulation.

cannot be applied to the globular case. The corresponding tip shape of the upstream arm at different time is imposed in the tip radius plotting diagram as shown in Figure 7 . It is clear that, during the initial stage $(t<57.6 \mathrm{~s})$, the crystal exhibits globular shape, and the interfacial energy anisotropy 


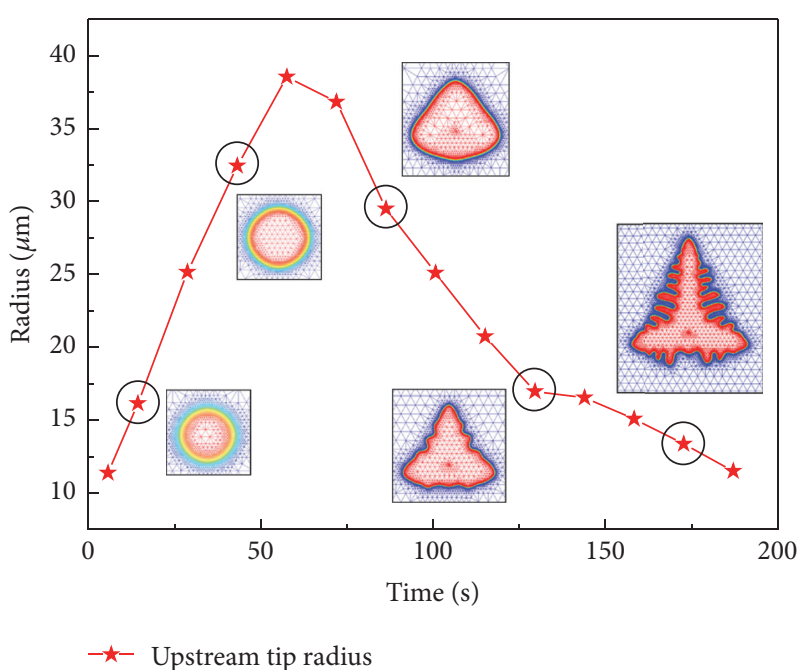

FIGURE 7: The corresponding crystal shape to the variation of the tip radius of the upstream arm.

has not yet effectively selected the preferred $\langle 100\rangle$ growth direction. At the time of $57.6 \mathrm{~s}$, the tip reaches its maximum upstream tip radius, and the growth anisotropy starts to play a dominant role. Then, the tip radius behaves in a normal way with the tip velocity in accordance with the theories of the dendrite. This phenomenon appears not only in the convective case, but also in the purely diffusive case [16]. The globular-to-dendritic transition is common in solidification, and the present computation further convinces us that phasefield simulation is an effective way to deal with this transition with the incorporation of the melt convection effects.

\subsection{Comparison of the Phase-Field Simulation with the In Situ} Observation. In order to examine the accuracy of the convective phase-field simulation, the calculated dendrite growth is compared with the in situ and real-time experimental observations on Al- $4 \mathrm{wt} . \% \mathrm{Cu}$ alloy [16]. The samples are $40 \mathrm{~mm}$ in length, $6 \mathrm{~mm}$ in width, and about $200 \mu \mathrm{m}$ in thickness. Since the thickness is sufficiently smaller than length and width, the samples can be roughly regarded as two-dimensional. The processing conditions of the experiment are the same as those adopted in the phase-field model in Table 1. One of the radiographs imaging equiaxed dendritic crystals is shown in Figure 8. In the experiment, the sedimentation of some dendrites occurred during solidification but was not observed apparently as they moved a very short distance. The relatively large density of crystals at the lower part of the sample is because these crystals nucleated at earlier time than those at the upper part. For more detailed description of this experiment, refer to [16]. Two dendrites are selected, labeled as D1 and D2. Considering that D1 has an almost perfect fourfold symmetry, it is selected first for study. Unfortunately, the measured growth velocity shows that once D1 is observed by the naked eyes in the radiograph, it is already poisoned by the surrounding grains [16]. This is because the time and spatial resolutions used in the experiment observation limited the recording at the early stage of the morphology

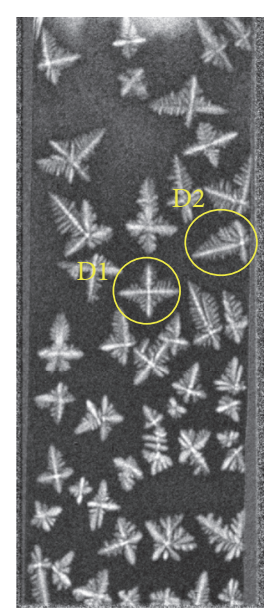

FIGURE 8: One selected sequential image recorded by means of synchrotron X-ray radiography [16]. The width of the image is $5.04 \mathrm{~mm}$. The selected dendrites are marked by yellow circles.

evolution of D1. Moreover, due to the solid blocking of melt convection and rejected solute from neighboring grains, D1 grew with being surrounded by an almost uniform solute field. Therefore, no obvious influence by natural convection is observed from the symmetric dendritic morphology. Hence, the dendrite D2 was then selected for comparison to the simulation data. As can be seen in Figure 8, the left perpendicular tip of D2 can grow into an unobstructed space for a long time without interaction with other dendrites after its nucleation, which is similar to the previous diffusive phasefield simulation [16].

Figure 9 shows the comparison of the tip velocities obtained from convective phase-field simulation with experimental measurements. In order to illustrate the effect of natural convection clearly on the growing speed of tips, the value of the purely diffusive phase-field simulation [16] is also plotted. Here, the $x$-coordinate is the external undercooling $\Delta T$, which is obtained using the formulation $\Delta T=R t$, and the $y$-coordinate is the tip velocity. Significant discrepancy between experiment and the purely diffusive phase-field model (PFM) exists as shown in Figure 9. As solidification continues, the discrepancy becomes larger and larger. The tip velocity measured in the experiment can even be one order of magnitude larger than the velocity predicted by the purely diffusive PFM. Since the dendritic arm tip on the left side which is almost perpendicular to the side wall is selected to measure the growth velocity in the experiment, the tip velocity of the perpendicular arm in the simulation coupling with natural convection (i.e., convective PFM) is chosen to compare with experimental data. As plotted in the Figure 9, the convective perpendicular tip velocity is almost twice the purely diffusive velocity. As a result, the discrepancy between the experiment and convective PFM is smaller than that between experiment and diffusive PFM. This comparison indicates that the natural convection indeed has an obvious effect on the dendritic growth. Moreover, the growth of D2 is not exactly in line with the gravity direction and surrounded by several dendrites. Therefore, the convection 
TABLE 1: The parameters used for phase-field simulation of equiaxed dendritic growth of Al-4 wt.\%Cu alloy with natural convection.

\begin{tabular}{lc}
\hline Solute partition coefficient, $k$ & 0.14 \\
Liquidus temperature, $T_{l}$ & $933.47 \mathrm{~K}$ \\
Solute diffusion coefficient in solid, $D_{s}$ & $1.15 \times 10^{-8} \mathrm{~cm}^{2} / \mathrm{s}$ \\
Solute diffusion coefficient in liquid, $D_{l}$ & $2.4 \times 10^{-5} \mathrm{~cm}^{2} / \mathrm{s}$ \\
Gibbs-Thomson coefficient, $\Gamma$ & $2.36 \times 10^{-5} \mathrm{~cm}^{\circ} \mathrm{K}$ \\
Liquidus slope, $m$ & $-3.5 \mathrm{~K} / \mathrm{wt} . \%$ \\
Initial concentration, $C_{0}$ & $4 \mathrm{wt} . \% \mathrm{Cu}$ \\
Interface width parameter, $\xi$ & 54 \\
Surface energy anisotropy strength, $\varepsilon_{4}$ & 0.0106 \\
Kinematic viscosity, $v$ & $5.0 \times 10^{-3} \mathrm{~cm}^{2} / \mathrm{s}$ \\
Density, $\rho$ & $2.45 \mathrm{~g} / \mathrm{cm}^{3}$ \\
Thermal expansion coefficient, $\alpha_{T}$ & $1.0 \times 10^{-4} \mathrm{k}^{-1}$ \\
Solutal expansion coefficient, $\alpha_{c}$ & $9.2 \times 10^{-3}(\mathrm{wt} . \%)^{-1}$ \\
Gravitational acceleration, $g$ & $-9.8 \times 10^{2} \mathrm{~cm}^{2} / \mathrm{s}^{2}$ \\
Dimensionless friction coefficient, $h$ & 2.757 \\
Cooling rate, $R$ & $0.5 \mathrm{~K} / \mathrm{min}^{2}$ \\
\hline
\end{tabular}

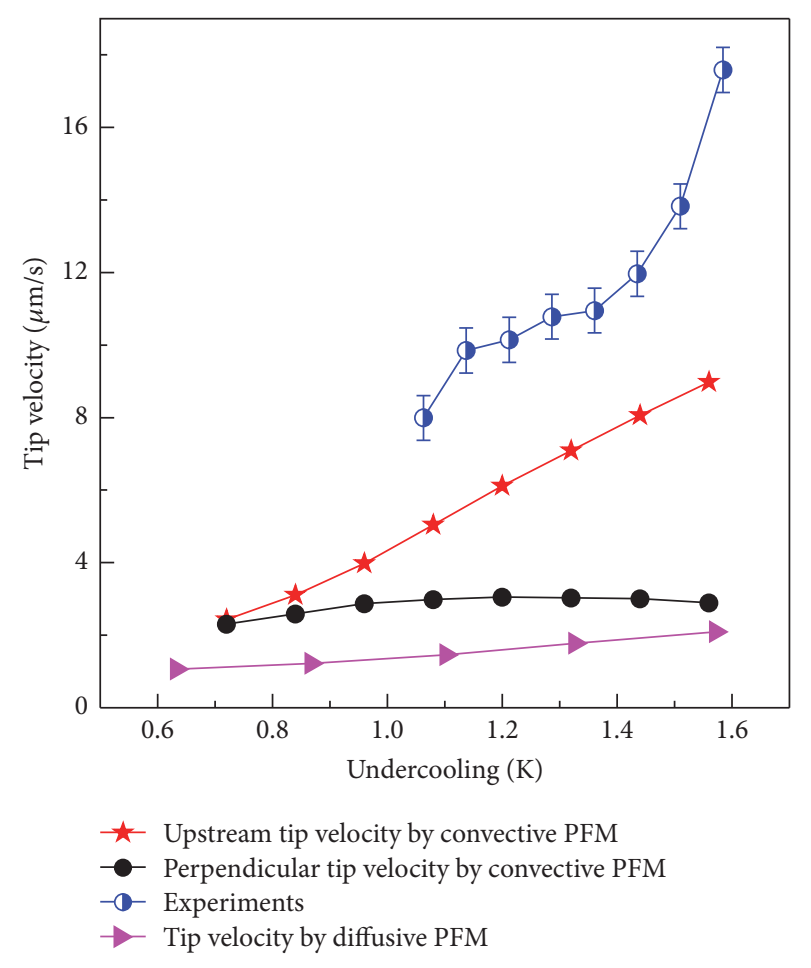

FIGURE 9: Comparison between experiment and phase-field simulation in the presence of natural convection. The phase-field simulation result without natural convection is also plotted.

pattern adjacent to D2 is definitely much more complex than simulation. The growth rate of the upstream tip in the convective PFM simulation is also plotted for comparison. Within our expectation, the velocity of the upstream tip is closer to the experiment. This correspondence should be attributed to the fact that the perpendicular tip in the experiment has a slightly downward orientation, and then the solutes are easily carried away by the flow. The environment of this downward perpendicular tip is similar to that of the upstream tip in the phase-field simulation. However, there still exists a little discrepancy between experimental data and prediction of the convective PFM. This may be due to other effects that are not taken into consideration, such as the sinking of the solid grain and 3D simulation. In the future work, these effects should be coupled for a more accurate prediction.

\section{Conclusion}

First, a quantitative 2D phase-field model incorporating fluid flow dynamics is employed to investigate the effect of natural convection on the dendritic growth from the melt of Al-4 wt.\%Cu alloy solidified by continuous coolingdown. The parameters of the model are adopted from the real synchrotron X-ray radiography experiment, and hence it is reasonable to link the phase-field simulation to the experiment. As expected, the simulation shows that natural convection induced by the gravity has an obvious effect on the morphology evolution of the equiaxed dendrite, leading to different tip velocities at different growth directions. The natural convection transports the solute rejected from the crystal downwards and redistributes the solute asymmetrically in the whole domain, which in turn causes the asymmetrical realistic undercooling at the four dendritic tips. As the dendrite grows, the impact of natural convection on dendrite evolution becomes more important due to the increment of convective strength. Finally, the quantitative comparison between experiments and simulations of convective and diffusive phasefield modeling reveals that natural convection plays a crucial role in the dendrite growth dynamics of metallic alloys on the earth. However, there are still some uncertainties required to be clarified, such as the difference between $2 \mathrm{D}$ and $3 \mathrm{D}$ simulations, the growth interaction between dendrites, and the flow pattern among many dendritic crystals driven by graded solute and temperature. 


\section{Competing Interests}

The authors declare that there are no competing interests regarding the publication of this paper.

\section{Acknowledgments}

This work is supported by National Natural Science Foundation for Young Scientists of China (Grant no. 51401223) and National Natural Science Foundation of China (Grant no. 51271184).

\section{References}

[1] W. Kurz and D. J. Fisher, Fundamentals of Solidification, CRC Press, New York, NY, USA, 1992.

[2] R. Trivedi and W. Kurz, "Dendritic growth," International Materials Reviews, vol. 39, no. 2, pp. 49-74, 1994.

[3] C. Y. Wang and C. Beckermann, "Equiaxed dendritic solidification with convection: part I. Multiscale/multiphase modeling," Metallurgical and Materials Transactions A, vol. 27, no. 9, pp. 2754-2764, 1996.

[4] C. Y. Wang and C. Beckermann, "Equiaxed dendritic solidification with convection: part II. Numerical simulations for an Al-4 Wt pct Cu alloy," Metallurgical and Materials Transactions A, vol. 27, no. 9, pp. 2765-2783, 1996.

[5] M. E. Glicksman, S. R. Coriell, and G. B. McFadden, "Interaction of flows with the crystal-melt interface," Annual Review of Fluid Mechanics, vol. 18, pp. 307-335, 1986.

[6] Y.-W. Lee, R. N. Smith, M. E. Glicksman, and M. B. Koss, "Effects of buoyancy on the growth of dendritic crystals," Annual Review of Heat Transfer, vol. 7, pp. 59-139, 1996.

[7] M. E. Glicksman, M. B. Koss, and E. A. Winsa, "Dendritic growth velocities in microgravity," Physical Review Letters, vol. 73, no. 4, pp. 573-576, 1994.

[8] M. E. Glicksman, M. B. Koss, L. T. Bushnell, J. C. Lacombe, and E. A. Winsa, "Dendritic growth of succinonitrile in terrestrial and microgravity conditions as a test of theory," ISIJ International, vol. 35, no. 6, pp. 604-610, 1995.

[9] M. B. Koss, L. T. Bushnell, J. C. LaCombe, and M. E. Glicksman, "The effect of convection on dendritic growth under microgravity conditions," Chemical Engineering Communications, vol. 15253, pp. 351-363, 1996.

[10] G. Ivantsov, "Temperature field around a spherical, cylindrical, and needle-shaped crystal, growing in a pre-cooled melt," Doklady Akademii Nauk SSSR, vol. 1, pp. 567-569, 1947.

[11] A. Bogno, H. Nguyen-Thi, N. Bergeon et al., "Application of synchrotron X-ray radiography to the study of dendritic equiaxed microstructure formation in Al-Cu alloys," Nuclear Instruments and Methods in Physics Research Section B, vol. 268, no. 3-4, pp. 394-398, 2010.

[12] A. Bogno, H. Nguyen-Thi, A. Buffet et al., "Analysis by synchrotron X-ray radiography of convection effects on the dynamic evolution of the solid-liquid interface and on solute distribution during the initial transient of solidification," Acta Materialia, vol. 59, no. 11, pp. 4356-4365, 2011.

[13] A. Bogno, H. Nguyen-Thi, G. Reinhart, B. Billia, and J. Baruchel, "Growth and interaction of dendritic equiaxed grains: in situ characterization by synchrotron X-ray radiography," Acta Materialia, vol. 61, no. 4, pp. 1303-1315, 2013.
[14] Y. Chen, A.-A. Bogno, B. Billia et al., "Phase-field modeling of the initial transient in directional solidification of $\mathrm{AI}-4 \mathrm{wt} \% \mathrm{Cu}$ alloy," ISIJ International, vol. 50, no. 12, pp. 1895-1900, 2010.

[15] Y. Chen, A.-A. Bogno, N. M. Xiao et al., "Quantitatively comparing phase-field modeling with direct real time observation by synchrotron X-ray radiography of the initial transient during directional solidification of an Al-Cu alloy," Acta Materialia, vol. 60, no. 1, pp. 199-207, 2012.

[16] Y. Chen, D. Z. Li, B. Billia, H. Nguyen-Thi, X. B. Qi, and N. M. Xiao, "Quantitative phase-field simulation of dendritic equiaxed growth and comparison with in situ observation on $\mathrm{Al}-4$ wt.\% $\mathrm{Cu}$ alloy by means of synchrotron X-ray radiography," ISIJ International, vol. 54, no. 2, pp. 445-451, 2014.

[17] N. Shevchenko, O. Roshchupkina, O. Sokolova, and S. Eckert, "The effect of natural and forced melt convection on dendritic solidification in Ga-In alloys," Journal of Crystal Growth, vol. 417, Article ID 22645, pp. 1-8, 2015.

[18] P. Bouissou and P. Pelcé, "Effect of a forced flow on dendritic growth," Physical Review A, vol. 40, no. 11, pp. 6673-6680, 1989.

[19] B. Cantor and A. Vogel, "Dendritic solidification and fluid flow," Journal of Crystal Growth, vol. 41, no. 1, pp. 109-123, 1977.

[20] R. Ananth and W. N. Gill, "Dendritic growth with thermal convection," Journal of Crystal Growth, vol. 91, no. 4, pp. 587598, 1988.

[21] R. Ananth and W. N. Gill, "Self-consistent theory of dendritic growth with convection," Journal of Crystal Growth, vol. 108, no. 1-2, pp. 173-189, 1991.

[22] C. Ming-Wen, W. Bao, and W. Zi-Dong, "Effect of buoyancydriven convection on steady state dendritic growth in a binary alloy," Chinese Physics B, vol. 22, no. 11, Article ID 116805, 2013.

[23] M. D. Dupouy, B. Drevet, and D. Camel, "Influence of convection on the selection of solidification microstructures at low growth rates," Journal of Crystal Growth, vol. 181, no. 1-2, pp. 145159, 1997.

[24] I. Steinbach, "Pattern formation in constrained dendritic growth with solutal buoyancy," Acta Materialia, vol. 57, no. 9, pp. 2640-2645, 2009.

[25] X. Tong, C. Beckermann, A. Karma, and Q. Li, "Phase-field simulations of dendritic crystal growth in a forced flow," Physical Review E, vol. 63, no. 6, Article ID 061601, 2001.

[26] C. C. Chen, Y. L. Tsai, and C. W. Lan, "Adaptive phase field simulation of dendritic crystal growth in a forced flow: $2 \mathrm{D}$ vs $3 \mathrm{D}$ morphologies," International Journal of Heat and Mass Transfer, vol. 52, no. 5-6, pp. 1158-1166, 2009.

[27] C. W. Lan and C. J. Shih, "Phase field simulation of nonisothermal free dendritic growth of a binary alloy in a forced flow," Journal of Crystal Growth, vol. 264, no. 1-3, pp. 472-482, 2004.

[28] C. W. Lan and C. J. Shih, "Efficient phase field simulation of a binary dendritic growth in a forced flow," Physical Review E, vol. 69, no. 3, Article ID 031601, 2004.

[29] M. Zhu, D. Sun, S. Pan, Q. Zhang, and D. Raabe, "Modelling of dendritic growth during alloy solidification under natural convection," Modelling and Simulation in Materials Science and Engineering, vol. 22, no. 3, Article ID 034006, 2014.

[30] E. Bänsch and A. Schmidt, "Simulation of dendritic crystal growth with thermal convection," Interfaces and Free Boundaries, vol. 2, no. 1, pp. 95-115, 2000.

[31] R. Tönhardt and G. Amberg, "Simulation of natural convection effects on succinonitrile crystals," Physical Review E, vol. 62, pp. 828-836, 2000. 
[32] P. Zhao, J. C. Heinrich, and D. R. Poirier, "Dendritic solidification of binary alloys with free and forced convection," International Journal for Numerical Methods in Fluids, vol. 49, no. 3, pp. 233-266, 2005.

[33] C. C. Chen and C. W. Lan, "Efficient adaptive three-dimensional phase field simulation of free dendritic growth under natural convection," Journal of Crystal Growth, vol. 312, no. 8, pp. 14371442, 2010.

[34] B. Echebarria, R. Folch, A. Karma, and M. Plapp, "Quantitative phase-field model of alloy solidification," Physical Review E, vol. 70, no. 6, Article ID 061604, 2004.

[35] A. Karma, "Phase-field formulation for quantitative modeling of alloy solidification," Physical Review Letters, vol. 87, no. 11, Article ID 115701, 2001.

[36] C. Beckermann, H. J. Diepers, I. Steinbach, A. Karma, and X. Tong, "Modeling melt convection inphase-field simulations of solidification," Journal of Computational Physics, vol. 154, no. 2, pp. 468-496, 1999.

[37] H.-J. Diepers, C. Beckermann, and I. Steinbach, "Simulation of convection and ripening in a binary alloy mush using the phasefield method," Acta Materialia, vol. 47, no. 13, pp. 3663-3678, 1999.

[38] A. Subhedar, I. Steinbach, and F. Varnik, "Modeling the flow in diffuse interface methods of solidification," Physical Review E, vol. 92, no. 2, Article ID 023303, 2015.

[39] R. Li, "On multi-mesh H-adaptive methods," Journal of Scientific Computing, vol. 24, no. 3, pp. 321-341, 2005.

[40] R. Li, http://dsec.pku.edu.cn/ rli/. 

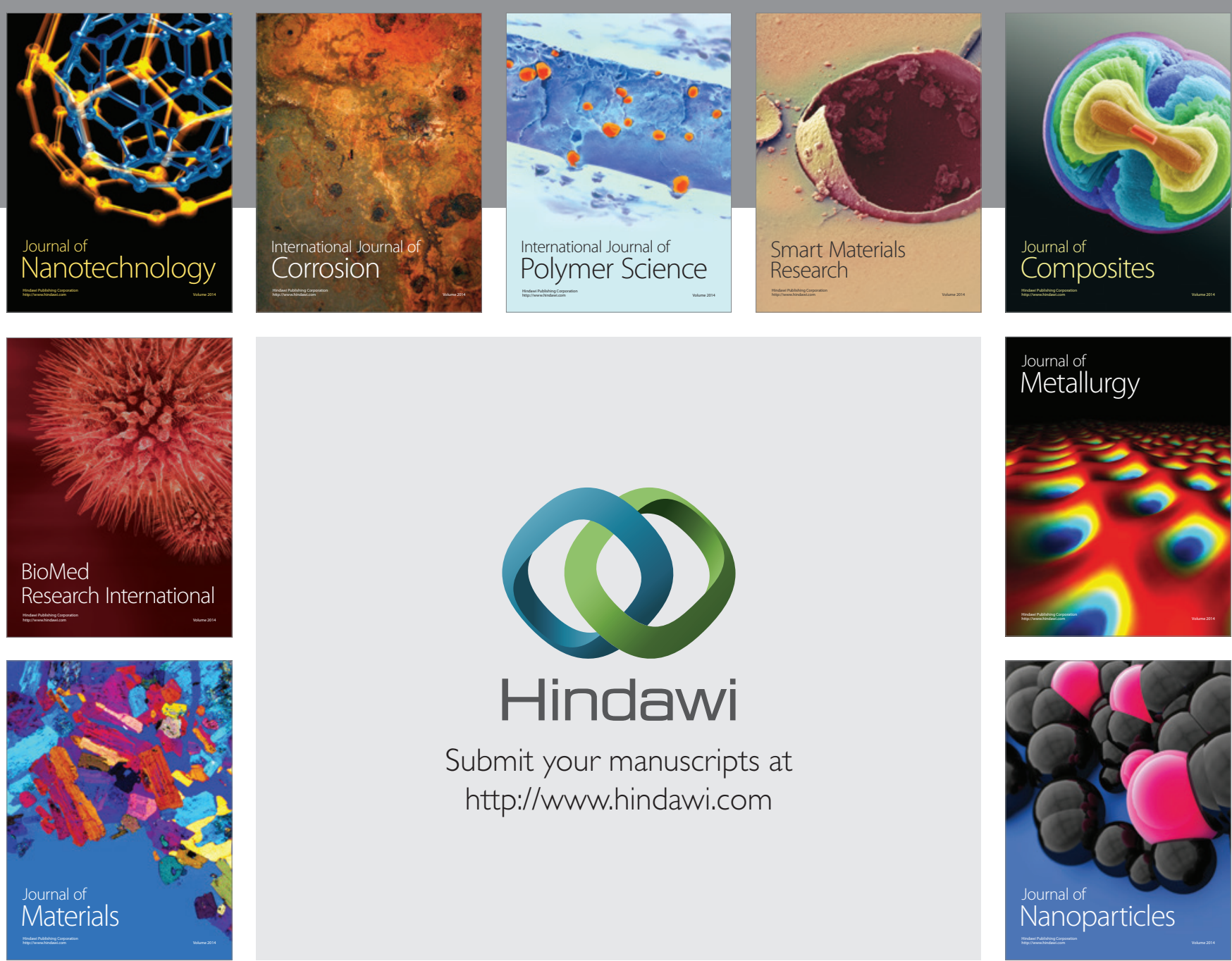

\section{Hindawi}

Submit your manuscripts at

http://www.hindawi.com

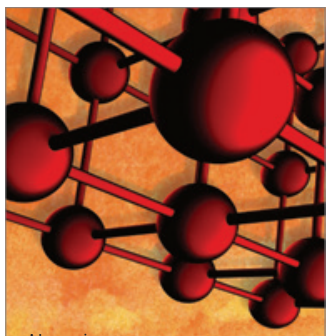

Materials Science and Engineering
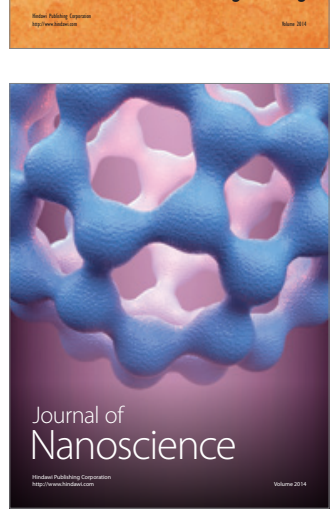
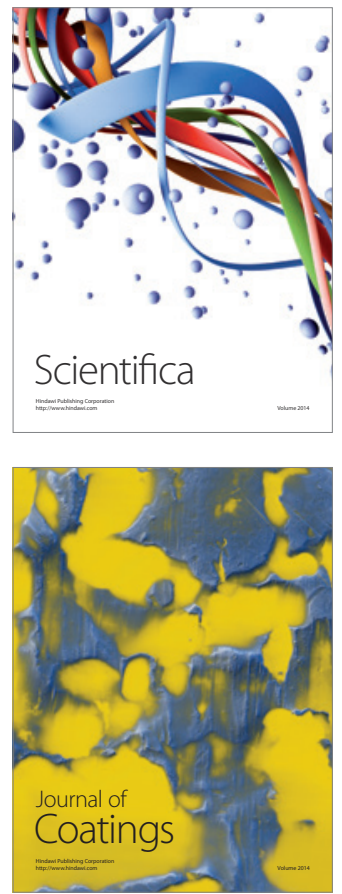
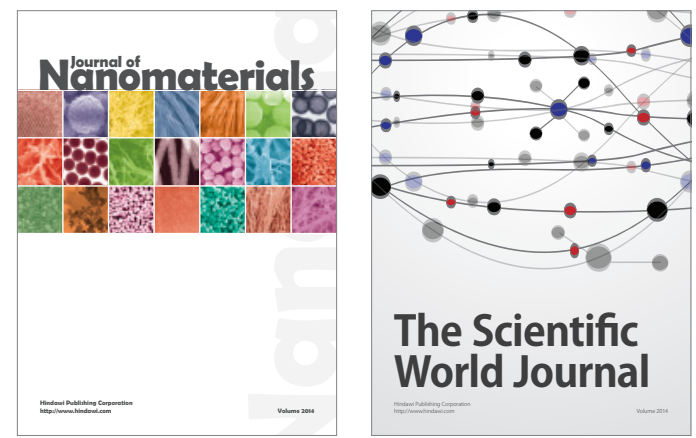

The Scientific World Journal
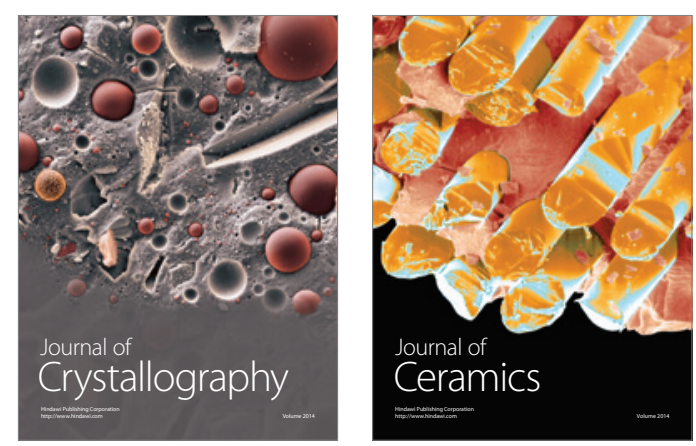
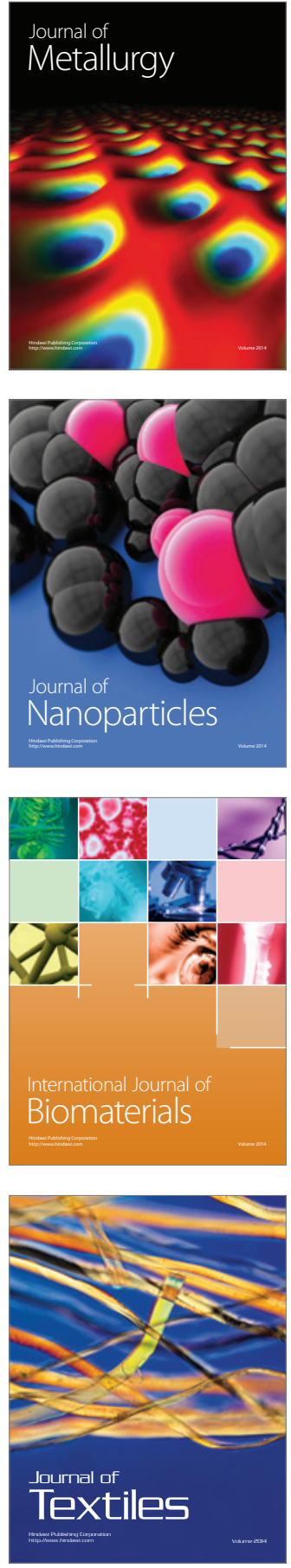